Trauma Berufskrankh 2008 10 [Suppl 3]:295-302

DOI 10.1007/s10039-008-1347-3

Online publiziert: 10. Mai 2008

(c) Springer Medizin Verlag 2008

\author{
M. Jaeger ${ }^{1}$ - D. Maier ${ }^{1} \cdot$ P. Ogon ${ }^{1,2} \cdot$ W. Köstler ${ }^{1} \cdot$ N.P. Südkamp \\ ${ }^{1}$ Department Orthopädie und Traumatologie, Universitätsklinikum Freiburg \\ 2 Sportorthopädische Gemeinschaftspraxis, Freiburg
}

\title{
Geschlossene und operative Therapieverfahren bei Schulterluxationen
}

sel-Band-Apparats, sehr häufig im Sinne einer Bankart-Läsion. Diese werden mit zunehmendem Alter seltener. Dafür treten insbesondere bei Rezidivinstabilitäten vermehrt Rupturen der Rotatorenmanschette auf. Von Loew et al. [22] wurden diese im Alter von 50-60 Jahren mit $<10 \%$ nach traumatischer Schultererstluxation und mit etwa $40 \%$ nach Rezidivluxationen angegeben. Ab dem 6o. Lebensjahr kommt es bei etwa 50\% nach Primärluxation und bei 70-100\% nach Rezidivluxation zu einer Ruptur der Rotatorenmanschette.

Hieraus lässt sich ableiten, dass konservative Therapieverfahren nicht immer zum gewünschten Erfolg führen. Operative Maßnahmen orientieren sich am Alter, der zugrunde liegenden Pathologie und dem funktionellen Anspruch des Patienten. Während junge Patienten in der Regel von einer Stabilisierung des ventralen Kapsel-Band-Apparats profitieren, empfiehlt sich bei älteren Patienten mit Ruptur eine Rekonstruktion der Rotatorenmanschette. In den letzten Jahren setzten sich hierbei zunehmend arthroskopische Therapieformen durch.

\section{Klassifikation}

Zur Einteilung von Schulterluxationen wurden diverse Klassifikationen vorgestellt. Keine von ihnen ist in der Lage, alle Mischformen der Schulterluxationen und -instabilitäten abzubilden. Im klinischen Alltag haben sich jedoch die Klassifikationen nach Matsen und Gerber bewährt.

\section{Matsen-Klassifikation}

Es werden eine atraumatische und eine traumatische Schulterinstabilität unterschieden [13]. Erstere lässt sich durch das Akronym AMBRII beschreiben, welches bedeutet, dass sich die Schulterinstabilität auf einen atraumatischen Ursprung (A) zurückführen lässt und in der Regel mit einer multidirektionalen Instabilität (M) verbunden ist. Häufig ist sie bilateral (B) vorhanden. Die Behandlung der atraumatischen Schulterinstabilität erfolgt in der Regel konservativ im Sinne einer Rehabilitation (R), wobei bei Beschwerdepersistenz eine operative Therapie, häufig in Form eines Verschlusses des Rotatorenintervalls (I) sowie einem inferioren Kapselshift (I), durchgeführt werden kann.

Die traumatische Schulterinstabilität wird durch das Akronym TUBS charakterisiert. Hier findet sich ein adäquates, traumatisches Ereignis (T), das in der Regel $\mathrm{zu}$ einer unidirektionalen Instabilität (U) führt. Häufig kommt es zu einer BankartLäsion (B). Insbesondere die chronische Form der traumatischen Schulterinstabilität wird chirurgisch (S) behandelt.

Obwohl die Matsen-Klassifikation das gesamte Spektrum der Instabilitätsformen

$\begin{aligned} & \text { Tab. } 1 \\
& \text { luxation nach konservativer Therapie }\end{aligned}$
\begin{tabular}{lll}
\hline Alter [Jahre] & Häufigkeit [\%] & Zitat \\
\hline$<20$ & $66-95$ & {$[29]$} \\
\hline$<30$ & $48-79$ & {$[14]$} \\
\hline$<40$ & $12-50$ & {$[14,29]$} \\
\hline
\end{tabular}




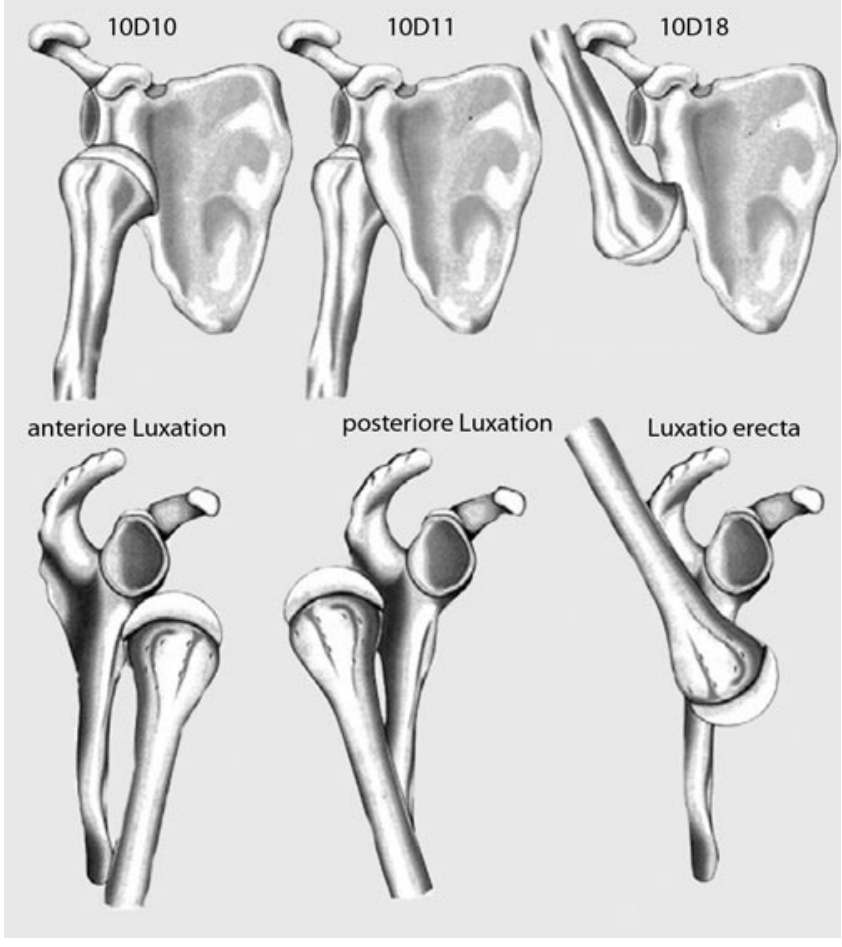

Abb. $1 \varangle$ Klassifikation der Schulterluxationen nach der Orthopaedic Trauma Association. (Nach [10]) und damit verbundenen Therapieoptionen umschreibt, ist sie sehr vereinfachend und berücksichtigt keine Mischformen, wie die Kombination einer traumatischen Schulterluxation bei vorbestehender Hyperlaxität.

\section{Gerber-Klassifikation}

Sie differenziert im Gegensatz zur Matsen-Klassifikation zwischen Instabilitäten und Hyperlaxitäten sowie indirekt zwischen traumatischen, d. h. unidirektionalen, und atraumatischen, d. h. multidirektionalen, Instabilitäten. 2002 wurde sie durch die ätiologische Beschreibung von 3 Schulterinstabilitätsklassen ergänzt ([10], - Tab. 2). Dabei erfassen die dynamischen Instabilitäten der Klasse B einen Großteil der klinisch relevanten Schulterinstabilitäten. Statische Instabilitäten der Klasse A können klinisch asymptomatisch sein und werden ggf. radiologisch im Rahmen einer relativen superioren, anterioren oder posterioren Verlagerung des Humeruskopfs diagnostiziert.

\section{Weitere Klassifikationen}

In der klinischen Praxis weniger gebräuchlich ist die Einteilung der Schulterinstabilitäten gemäß der Orthopaedic Trauma Association [10]. Der Kode 10-
D kennzeichnet dabei das Glenohumeralgelenk, der Buchstabe D die Luxation („dislocation“) und die Zahlenkombination $1 x$ die Luxationsrichtung. (• Tab. 3, - Abb. 1).

\section{Diagnostik}

Oft ist bereits die Klinik im Zusammenhang mit der Anamnese für die Schulterluxation pathognomonisch. Es findet sich regelhaft eine „leere Schultergelenkpfanne" mit Entrundung der Schultersilhouette, begleitet von erheblichen Schulterschmerzen und einer fixierten Bewegungseinschränkung.

Trotz dieser oft bereits klinisch eindeutigen Symptomatik hat sich eine suffiziente bildgebende Diagnostik anzuschließen. Als Standard gilt hier insbesondere bei traumatischen Schulterluxationen eine primäre, konventionelle Röntgenbildgebung in 2 Ebenen, um eine begleitende Luxationsfraktur und u. U. iatrogene Schädigungen auszuschließen. Bewährt hat sich neben einer echten a.-p. Projektion mit Darstellung des glenohumeralen Gelenkspalts die so genannte Skapula-Y-Aufnahme, die auch bei schmerzhaften Zuständen gut durchführbar ist. Eine transthorakale Aufnahmetechnik gilt heute vor dem Hintergrund der schlech- ten Beurteilbarkeit und der vergleichbar hohen Strahlenbelastung als obsolet.

Unmittelbar nach erfolgter Reposition ist die regelrechte glenohumerale Artikulation radiologisch nachzuweisen. Hierbei ist auf eine echte a.-p. Einstellung mit eindeutigem, überlappungsfreiem Nachweis des glenohumeralen Gelenkspalts zu achten, da nur so eine fortbestehende Luxation sicher ausgeschlossen werden kann (• Abb. 2).

Des Weiteren haben sich der Reposition erneut die Überprüfung der peripheren Durchblutung [31], Motorik und Sensibilität sowohl im Bereich der Hand als auch des Versorgungsgebiets des N. axillaris anzuschließen.

Zur weiteren Therapieplanung empfiehlt sich in der Folge elektiv die Vervollständigung der bildgebenden Diagnostik. Bei Vorliegen von knöchernen Pfannenrandfrakturen gelingt es mit einer CT, das Ausmaß der Fraktur präoperativ exakt zu bestimmen und die Technik der Osteosynthese in Abhängigkeit von der Fragmentgröße zu planen. Darüber hinaus lassen sich im CT präoperativ die Grenzen der Möglichkeit einer arthroskopischen Refixierung genau abschätzen. Auch bei größeren Hill-Sachs-Läsionen ist die CT von großem Wert, da sich die Größe und Lage der Impressionsfraktur exakt bestimmen und die weiteren operativen Therapieoptionen (Defektauffüllung, Rotationsosteotomie oder endoprothetische Versorgung) planen lassen. Während sich Frakturen des Tuberculum majus im Sinne eines knöchernen Ausrisses der Supraspinatussehne konventionell radiologisch gut abbilden lassen, werden knöcherne Abrissfrakturen des Tuberculum minus nach posteriorer Luxation konventionell radiologisch leicht übersehen. Auch hier ist die CT deutlich überlegen und bei klinischem Verdacht indiziert.

Empfehlenswert ist ferner eine MRT der verletzten Schulter [34]. Hier zeigen sich in Abhängigkeit vom Alter die Art der ventralen Kapsel-Band-Läsion und das Ausmaß der Luxationstasche. Hilfreich ist die MRT ferner, um Begleitverletzungen, insbesondere des Bizepssehnenankers oder der Rotatorenmanschette, darzustellen.

Während die Sonographie weit verbreitet und leicht durchführbar ist, ist die Ar- 


\begin{tabular}{|c|c|c|}
\hline Klasse & Subklasse & Charakteristika \\
\hline \multirow[t]{5}{*}{ A } & \multicolumn{2}{|c|}{ Statische Instabilitäten } \\
\hline & $\mathrm{A} 1$ & Superior \\
\hline & A2 & Anterior \\
\hline & $\mathrm{A} 3$ & Posterior \\
\hline & A4 & Inferior \\
\hline \multirow[t]{7}{*}{ B } & \multicolumn{2}{|c|}{ Dynamische Instabilitäten } \\
\hline & B1 & Chronisch verhakt \\
\hline & B2 & $\begin{array}{l}\text { Unidirektional, } \\
\text { ohne Hyperlaxität }\end{array}$ \\
\hline & B3 & $\begin{array}{l}\text { Unidirektional, } \\
\text { multidirektionale } \\
\text { Hyperlaxität }\end{array}$ \\
\hline & B4 & $\begin{array}{l}\text { Multidirektional, } \\
\text { ohne Hyperlaxität }\end{array}$ \\
\hline & B5 & $\begin{array}{l}\text { Multidirektional, } \\
\text { multidirektionale } \\
\text { Hyperlaxität }\end{array}$ \\
\hline & B6 & Willkürlich \\
\hline C & \multicolumn{2}{|c|}{ Willkürliche Luxationen } \\
\hline
\end{tabular}

Tab. 3 Klassifikation der Schulterlu-

xationen nach der Orthopaedic Trauma

Association. (Nach [10])

\begin{tabular}{cl} 
10-D10 & $\begin{array}{l}\text { Glenohumerale Luxation nach } \\
\text { anterior }\end{array}$ \\
\hline $10-D 11$ & $\begin{array}{l}\text { Glenohumerale Luxation nach } \\
\text { posterior }\end{array}$ \\
10-D18 & $\begin{array}{l}\text { Glenohumerale Luxation nach } \\
\text { inferior (Luxatio erecta) }\end{array}$ \\
\hline
\end{tabular}

thrographie im Zeitalter des MRT deutlich in den Hintergrund getreten. Um bei besonderen Fragestellungen, insbesondere bei Beurteilung des Labrums und des Bizepssehnenankers, die Wahrscheinlichkeit falsch-negativer Befunde zu verringern, wird eine indirekte Kontrastierung mit Kontrastmittel empfohlen [1].

\section{Therapie}

\section{Geschlossene, konservative Therapie der posttraumatischen Schulterinstabilität}

Zur Reposition einer Schulterluxation sind viele Techniken gebräuchlich. Weit verbreitetet sind immer noch die Repositionstechniken nach Arlt und Hippokrates. Sie zeichnen sich dadurch aus, dass mitunter große Kraftanstrengungen notwendig sind, um eine Reposition zu erzielen. Teilweise gelingt dies nur in Analgosedierung, um die schmerzbedingte

Trauma Berufskrankh 2008 10 [Suppl 3]:295-302 DOI 10.1007/s10039-008-1347-3

(C) Springer Medizin Verlag 2008

\section{Jaeger · D. Maier · P. Ogon · W. Köstler · N.P. Südkamp Geschlossene und operative Therapieverfahren bei Schulterluxationen}

\section{Zusammenfassung}

Schulterluxationen sind ein häufiges Ereignis. Insbesondere bei jungen Männern muss von einer Rezidivhäufigkeit nach konservativer Therapie von etwa $87 \%$ innerhalb der ersten 2 Jahre nach dem Trauma ausgegangen werden. Eine posttraumatische Immobilisation in Außenrotationsstellung scheint dabei günstigere Ergebnisse zu liefern. Zur Reposition werden schonende Verfahren, z. B. die Technik nach Stimson empfohlen, die in Bauchlage des Patienten $u$. U. unter Zuhilfenahme einer Skapulamanipulation eine sichere Reposition ermöglicht. Die operative Therapie richtet sich nach der zugrunde liegenden Pathologie, dem Alter und dem Anspruch des Patienten. Die offene Bankart-Operation gilt in der Literatur bezüglich der Reluxationsra-

\section{Closed and surgical treatment of shoulder dislocation}

\section{Abstract}

Shoulder dislocations are common. Young male patients especially have a very high risk of persistent or recurrent instability (about $87 \%$ ) within the first 2 years after nonoperative treatment following trauma. Posttraumatic immobilisation in external rotation might be a better option to prevent redislocation. We recommend a gentle method of reduction, such as Stimson's technique, which can be performed by manipulating the scapula with the patient in the prone position. Surgical treatment needs to be adapted to the underlying pathology, the patient's age, and the patient's individual demands. According to the literature, the gold-standard technique for refixation of a torn capsu- te nach wie vor als Goldstandard zur Refixierung des abgerissenen ventralen Kapsel-Labrum-Komplexes. In der Hand des Geübten scheint jedoch auch eine arthroskopische Refixierung legitimiert, da sie insbesondere sehr gute funktionelle Ergebnisse erwarten lässt. In den letzten Jahren haben sich deren Möglichkeiten stetig verbessert. Unter Verwendung von knotenlosen Ankersystemen sind mittlerweile auch arthroskopische Kapselshifts zuverlässig durchführbar.

\section{Schlüsselwörter}

Schulterluxation - Konservative Therapie . Bankart-Läsion · Arthroskopische

Stabilisierung $\cdot$ Kapselshift lar-labrum complex, with regard to redislocation rate, is still the open Bankart procedure. However, in the hands of an experienced surgeon, the results of arthroscopic stabilisation seem very promising; the functional results especially appear to be superior to open techniques. Over the last years, arthroscopic fixation techniques have improved steadily. Using knotless anchors, it is now possible to perform sufficient arthroscopic capsular shifts.

\section{Keywords}

Shoulder dislocation - Nonoperative treatment - Bankart lesion - Arthroscopic stabilisation · Capsular shift 

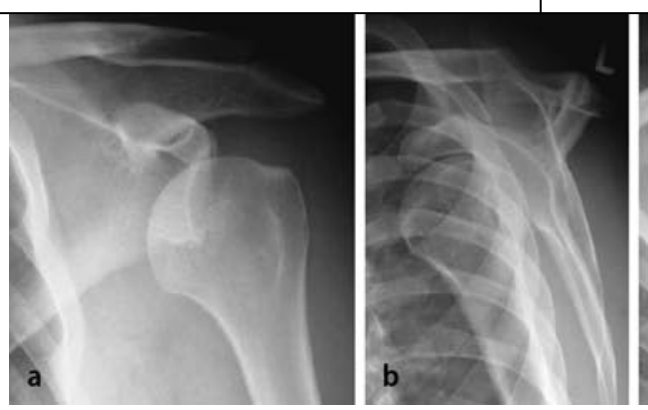

\section{Verletzungen und Verletzungsfolgen am Schultergelenk}

Abb. $2 \Delta$ Konventionelle radiologische Diagnostik einer ventralen glenohumeralen Luxation vor $(\mathbf{a}, \mathbf{b})$ und nach Reposition (c-e), deutlicher Unterschied zwischen der normalen (c) und der wahren a.-p. Aufnahme (d)
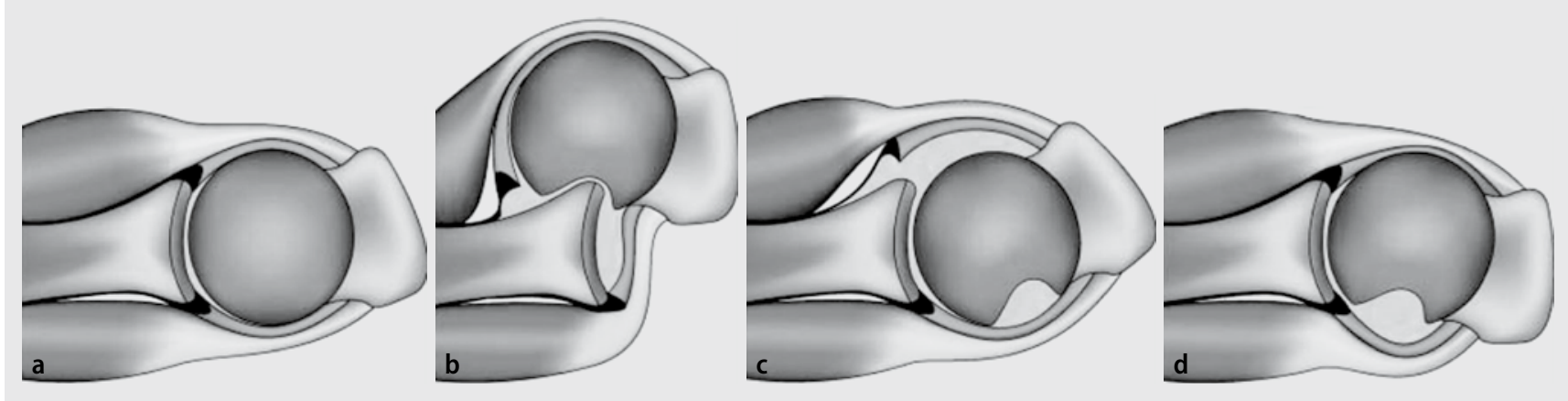

Abb. $3 \Delta$ a,b Traumatische Erstluxation mit Ausbildung einer Labrum-Ligament-Läsion, c Medialisierung des Labrum-Ligament-Komplexes durch Ruhigstellung der Schulter in Innenrotation, $\mathbf{d}$ Lateralisierung und somit Reposition des Labrum-Ligament-Komplexes auf den Glenoidrand durch Anspannung der ventralen Kapsel und des M. subscapularis in Außenrotation. (Aus [32])

Abwehrspannung zu überwinden. Diese Technik kann insbesondere bei osteoporotischer Knochenqualität zu iatrogenen Frakturen führen.

Weniger verbreitet ist die schonende Methode nach Stimson [33], in der sich der Patient in Bauchlage befindet. In Verbindung mit einer Skapulamanipulation lässt sich auch ohne Extensionsgewicht eine Reposition zuverlässig erzielen. Die in dieser Technik erreichbare Erfolgsquote liegt initial bei $90,2 \%$ ohne begleitende Medikation und bei 100\% im Zweitversuch.[3]

Kontrovers werden die Notwendigkeit und die Art der Immobilisation post repositionem diskutiert. Üblich ist eine primäre Ruhigstellung in einem Gilchrist-Verband, wobei sich die betroffene Schulter in Innenrotation befindet. Dabei scheint die Dauer der Immobilisation keinen Einfluss auf die Rezidivrate zu haben [15], sodass eine kurzfristige, schmerzadaptierte Ruhigstellung, gefolgt von einer frühfunktionellen Nachbehandlung, empfohlen wird.

Im Gegensatz dazu scheint eine Immobilisation in Außenrotationsstellung die Reluxationsrate günstig zu beeinflus- sen. Itoi et al. [17] konnten diesbezüglich nachweisen, dass sich ab $o^{\circ}$ mit zunehmender Außenrotation der ventrale Labrum-Kapsel-Band-Apparat straff an das Glenoid anlegt und somit eine stabilere Ausheilung begünstigt (- Abb. 3). MRTkontrollierte Studien sowie erste klinische Ergebnisse sind Erfolg versprechend [18, 32].

Unabhängig von der Art der Schulterluxation sind in der Literatur aufgrund der oben angeführten epidemiologischen Daten die in 0 Tab. 4 beschriebenen Indikationen zur konservativen Therapie allgemein akzeptiert:

\section{Operative Therapieverfahren}

Ihre Form richtet sich insbesondere nach der zugrunde liegenden Pathologie. Diese kann betreffen:

- das Glenoid,

- das Labrum,

- den Kapsel-Band-Apparat,

- die Rotatorenmanschette oder

- den Humerus.

Für die Indikationsstellung sind jedoch neben der exakten Diagnose und Klas- sifikation der glenohumeralen Luxation verschiedene weitere Gesichtspunkte entscheidend. Diese umfassen:

- mögliche Begleitverletzungen,

- das Alter des Patienten,

- seine Funktionsansprüche und Mitarbeit (Compliance).

Eine echte Notfallsituation im Rahmen einer glenohumeralen Luxation, die ein unmittelbares, operatives Vorgehen erfordert, wird selten beobachtet. Sie kann sich im Rahmen von begleitenden Gefäßund Nervenverletzungen ergeben. Aber auch gewisse Formen der Luxationsfrakturen oder verhakte, geschlossen nicht reponierbare Luxationen können ein rasches operatives Vorgehen notwendig werden lassen.

Ansonsten stellen Schultergelenk stabilisierende Operationen elektiv durchzuführende Eingriffe dar. Im Allgemeinen sind die in $\square$ Tab. 5 aufgeführten Operationsindikationen akzeptiert.

\section{Glenoidfraktur}

Begleitende Glenoidfrakturen imponieren typischerweise als Pfannenrandfrakturen. In der Hand des Geübten lassen sie 
sich sehr gut arthroskopisch reponieren und refixieren. $A b$ einer Größe von mehr als 1/5 der kaudalen Glenoidfläche wird jedoch allgemein ein offenes Vorgehen angeraten. Nach erfolgter offener Reposition des Fragments über einen vorderen $\mathrm{Zu}$ gang bieten sich zur Osteosynthese Kleinfragmentschrauben oder vorzugsweise resorbierbare Polypins an (• Abb.4).

\section{Frakturen des Tuberculum majus}

Eine deutliche Dislokation des Tuberculum majus mit insbesondere Kranialisierung desselben um mehr als $5 \mathrm{~mm}$ sollte unserer Erfahrung nach reponiert und osteosynthetisch versorgt werden. Anderenfalls ist mit einem sekundären Impingement sowie einem funktionellen Defizit der Rotatorenmanschette zu rechnen. Hierbei hat es sich als vorteilhaft erwiesen, dass nach erfolgter übungsstabiler Osteosynthese die betroffene Schulter deutlich früher intensiv funktionell aktiv und passiv beübt werden kann. Zur Osteosynthese eignen sich vorzugsweise Kleinfragmentschrauben, die sowohl offen als auch perkutan eingebracht werden können.

\section{Frakturen des Tuberculum minus}

Sie werden häufig in der primär konventionellen Bildgebung übersehen, stellen jedoch eine absolute Operationsindikation dar. Sie sind als knöcherner Ausriss der Subskapularissehne zu interpretieren. Konservativ therapiert sind sie regelhaft von einem deutlichen funktionellen, insbesondere die Innenrotation betreffenden Funktionsdefizit begleitet. Auch hier empfiehlt sich in aller Regel eine Kleinfragmentschraubenosteosynthese.

\section{Hill-Sachs-Läsionen}

Sie lassen sich nach Calandra et al. [5] in 3 Stufen graduieren ( $\bullet$ Tab. 6).

Im Allgemeinen finden sie sich bei anterior-inferioren Schulterluxationen an loco typico, d. h. postero-superior am Humeruskopf, und bedürfen in aller Regel keiner speziellen Therapie. Bei Impressionen, die mehr als 1/3 der Zirkumferenz des Humeruskopfs ausmachen, ist jedoch von einer biomechanischen Wirksamkeit im Sinne einer vorderen Instabilität auszugehen, wobei sich der Humeruskopf im Rahmen eines ,engaging “ Hill-Sachs-Defekts aus dem Glenoid heraushebeln kann.
Tab. 4 Indikationen zur konservativen Therapie. (Nach [11, 12])

Kinder (meist atraumatische Luxation)

Jugendliche mit offenen Epiphysenfugen

Patienten im Alter zwischen 18 und 30 Ohne Hill-Sachs-Defekt

Jahren, wenn einer oder mehrere der Ohne knöcherne Bankart-Läsion

neben stehenden Faktoren vorliegen

Ohne adäquates Trauma

Ohne sportlichen Anspruch

Mit begleitender Kapsellaxität

Mit begleitender Axillarisschädigung

Mit muskulär willkürlicher/unwillkürlicher Dysbalance

Im Alter über 30 Jahre ohne knöcherne Bankart-Fraktur

Im Alter über 40 Jahre ohne Rotatorenmanschettenruptur

Tab. 5 Operationsindikationen im Rahmen einer traumatischen glenohumeralen Erstluxation

Absolute Operations- Irreponierbare Luxation mit interponiertem Weichteilgewebe

indikationen Knöcherne Bankart-Läsionen jenseits des kritischen Werts von 1/5 der

kaudalen Glenoidfläche (d. h. größer als $15 \mathrm{~mm}$ Länge und $5 \mathrm{~mm}$ Breite)

Mehr als $5 \mathrm{~mm}$ dislozierte Fraktur des Tuberculum majus

Begleitende Ruptur des Subskapularissehne

Begleitende Rotatorenmanschettenruptur beim älteren Patienten

Begleitende instabile Mehrfragmentfraktur des Humeruskopfs im Sinne einer Luxationsfraktur

Relative Operations- Alter zwischen 16 und 30 Jahre

indikationen Hoher Funktionsanspruch

Adäquates Trauma ohne Selbstreposition

Bankart- und Hill-Sachs-Läsion

Ausschluss einer Hyperlaxität

Gute Compliance

Tab. 6 Calandra-Klassifikation der Defektgröße einer Hill-Sachs-Läsion. (Nach [5])

\section{Grad Defektgröße}

I Defekt der Gelenkfläche ohne Beteiligung des subchondralen Knochens

II Defekt der Gelenkfläche mit Beteiligung des subchondralen Knochens

III Großer Defekt des subchondralen Knochens

Tab. 7 Rezidivraten nach arthroskopischer Stabilisierung einer posttraumatischen ventralen Schulterinstabilität mit Fadenankern

\begin{tabular}{|c|c|c|c|c|c|c|}
\hline Autor & Jahr & $\begin{array}{l}\text { Studien- } \\
\text { design }\end{array}$ & $\begin{array}{l}\text { Follow-up } \\
\text { [Monate] }\end{array}$ & $\begin{array}{l}\text { Pati- } \\
\text { enten }\end{array}$ & Technik & $\begin{array}{l}\text { Rezidive } \\
\text { [\% der Fälle] }\end{array}$ \\
\hline $\begin{array}{l}\text { Bottoni } \\
\text { et al. [4] }\end{array}$ & 2006 & $\begin{array}{l}\text { Prospektiv } \\
\text { randomisiert }\end{array}$ & 32 & $\begin{array}{l}29 \\
32\end{array}$ & $\begin{array}{l}\text { Offen vs. Arthrosko- } \\
\text { pisch (Fadenanker) }\end{array}$ & $\begin{array}{l}0 \\
0\end{array}$ \\
\hline $\begin{array}{l}\text { Carreira } \\
\text { et al. [6] }\end{array}$ & 2006 & Prospektiv & 48 & 85 & $\begin{array}{l}\text { Arthroskopisch } \\
\text { (Fadenanker) }\end{array}$ & 5 \\
\hline $\begin{array}{l}\text { Marquardt } \\
\text { et al. [23] }\end{array}$ & 2006 & Prospektiv & 42 & 54 & $\begin{array}{l}\text { Arthroskopisch } \\
\text { (Fadenanker) }\end{array}$ & 7,5 \\
\hline $\begin{array}{l}\text { Tjoumakaris } \\
\text { et al. [37] }\end{array}$ & 2006 & Retrospektiv & $\begin{array}{l}56 \\
40\end{array}$ & $\begin{array}{l}24 \\
69\end{array}$ & $\begin{array}{l}\text { Offen vs. Arthrosko- } \\
\text { pisch (Fadenanker) }\end{array}$ & $\begin{array}{l}4 \\
1\end{array}$ \\
\hline $\begin{array}{l}\text { Fabbriciani } \\
\text { et al. [8] }\end{array}$ & 2004 & $\begin{array}{l}\text { Prospektiv } \\
\text { randomisiert }\end{array}$ & 24 & $\begin{array}{l}30 \\
30\end{array}$ & $\begin{array}{l}\text { Offen vs. Arthrosko- } \\
\text { pisch (Fadenanker) }\end{array}$ & $\begin{array}{l}0 \\
0\end{array}$ \\
\hline $\begin{array}{l}\text { Ide et al. } \\
\text { [16] }\end{array}$ & 2004 & Prospektiv & 42 & 55 & $\begin{array}{l}\text { Arthroskopisch } \\
\text { (Fadenanker) }\end{array}$ & 7 \\
\hline $\begin{array}{l}\text { Kim et al. } \\
{[20]}\end{array}$ & 2002 & Prospektiv & 39 & $\begin{array}{l}30 \\
59\end{array}$ & $\begin{array}{l}\text { Offen vs. Arthrosko- } \\
\text { pisch (Fadenanker) }\end{array}$ & $\begin{array}{l}6,7 \\
3,4\end{array}$ \\
\hline
\end{tabular}



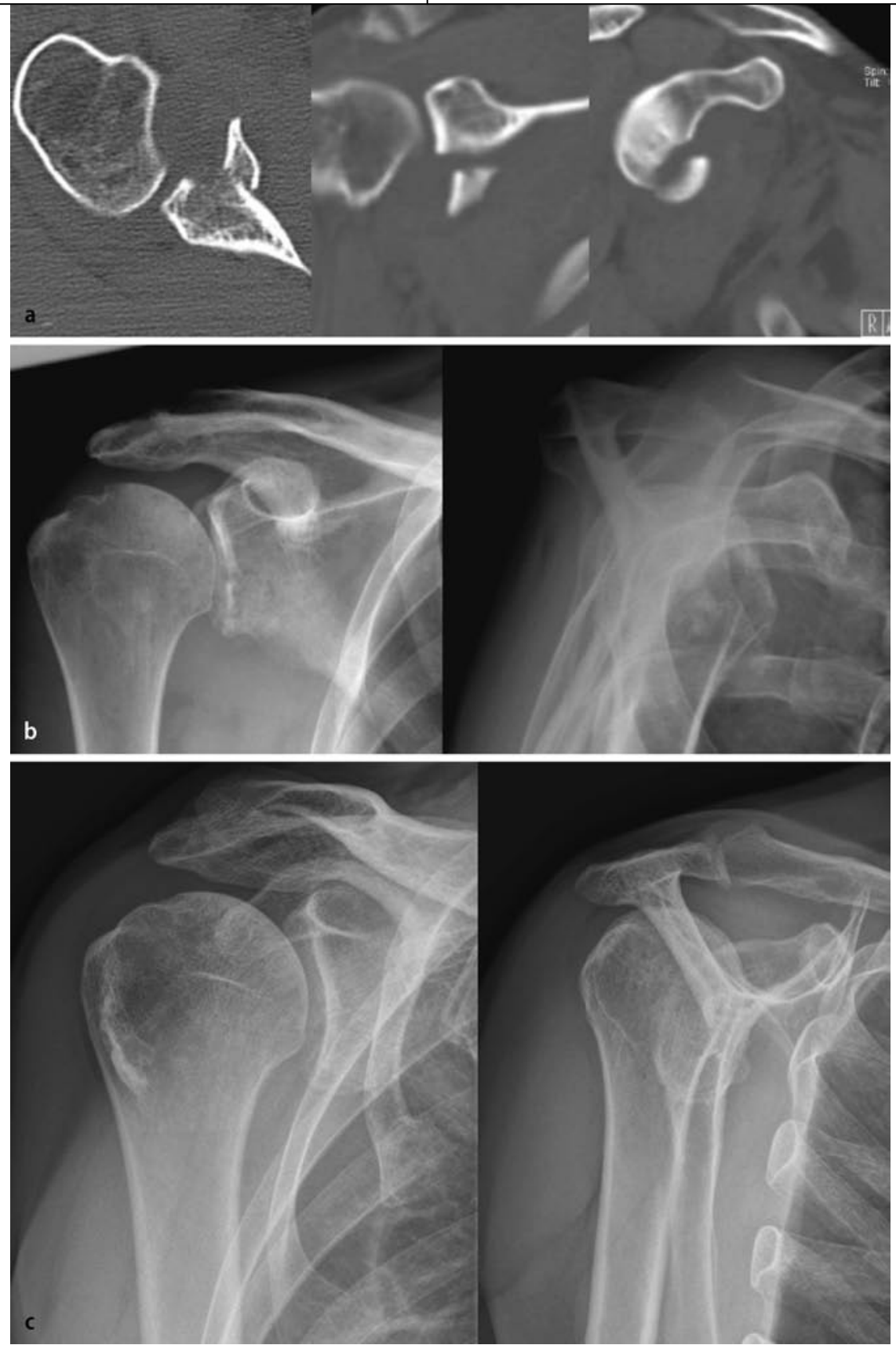

Abb. 4 \ Ventrale Schulterluxation mit großem knöchernem Bankart-Fragment vor (a), unmittelbar nach (b) und 4 Jahre nach Osteosynthese mit Polypins (c)

Insbesondere bei der hinteren Schulterluxation spielt das Vorhandensein einer „reversed" Hill-Sachs-Läsion hinsichtlich des Risikos einer Rezidivluxation eine wichtige Rolle.

Abhängig von der Defektgröße empfiehlt sich ein operatives Vorgehen. Während kleine Impressionen mit einem Impressionsausmaß von unter $20 \%$ der Gelenkfläche gut einer konservativen Therapie zugeführt werden können, sollte bei mittleren Impressionen von $20-40 \%$ der Gelenkfläche im Rahmen eines frühelek- tiven Eingriffs ein Aufstößeln der imprimierten Humeruskopfanteile erfolgen. Dies kann unter arthroskopischer Kontrolle durchgeführt werden. Alternativ besteht die Möglichkeit einer Rotationsosteotomie des Humerus, z. B. in der Technik nach Weber oder Saha. Ziel dieser Maßnahme ist es, den Humerusschaft gegenüber dem Humeruskopf zu rotieren, sodass bei erneuter Innenrotation des Arms die Impression nicht mehr im Bereich des hinteren Glenoidrands einhakt. Alternativ kann bei großen Impressionen von über
$40 \%$ der Gelenkfläche ein endoprothetischer Ersatz erwogen werden.

\section{Rupturen der Rotatorenmanschette}

Im Rahmen einer Schulterluxation sind sie insbesondere bei Patienten jenseits des 40. Lebensjahrs zu beobachten. Zu ihrer Versorgung haben sich zunehmend arthroskopische Verfahren etabliert. Große Defekte der Manschette sowie Rupturen der Subskapularissehne werden nach wie vor vorwiegend offen therapiert.

Im Rahmen eines offenen Vorgehens werden Läsionen im Bereich der Sehne des Supra- und Infraspinatusmuskels über einen lateralen Zugang mit Deltoidsplit erreicht. Hierbei ist darauf zu achten, dass die Inzision und der Zugang nicht weiter als $5 \mathrm{~cm}$ kaudal der lateralen Akromionkante reichen, um den $\mathrm{N}$. axillaris zu schützen. Läsionen im Bereich des Rotatorenintervalls, des Pulley-Komplexes und des M. subscapularis werden dagegen über einen ventralen, deltoideopektoralen Zugang versorgt.

\section{Verletzung des Kapsel-Labrum-Komplexes}

Beim jüngeren Patienten kommt es häufig zu einer Verletzung des Kapsel-Labrum-Komplexes, die offen oder aber auch arthroskopisch stabilisiert werden kann. Ziel der Operation ist es, ein Verheilen des abgerissenen Kapsel-Labrum-Komplexes in orthotoper Position zu ermöglichen.

Im Fall einer Reluxation ist davon auszugehen, dass ein instabiles Schultergelenk vorliegt, das spätestens dann einer Operation zugeführt werden sollte. Ansonsten kann es durch weitere Luxationen zu einer plastischen Deformierung und somit einer zunehmenden Ausweitung des Kapsel-Band-Apparats kommen. Die kritische Anzahl wird mit 5 Rezidiven angegeben.

Einer 2001 in Deutschland an 210 unfallchirurgischen Abteilungen durchgeführten Umfrage zur Folge wird als Verfahren der Wahl die Bankart-Operation durchgeführt [36]. Sie wurde bereits 1906 von Perthes [27] entwickelt und fand durch Bankart [2] 1926 weitere Verbreitung. Sie besteht im Wesentlichen in einer transossären Nahtrefixierung des abgetrennten Kapsel-Labrum-Komplexes auf Höhe der Gelenkfläche. Vereinfacht 

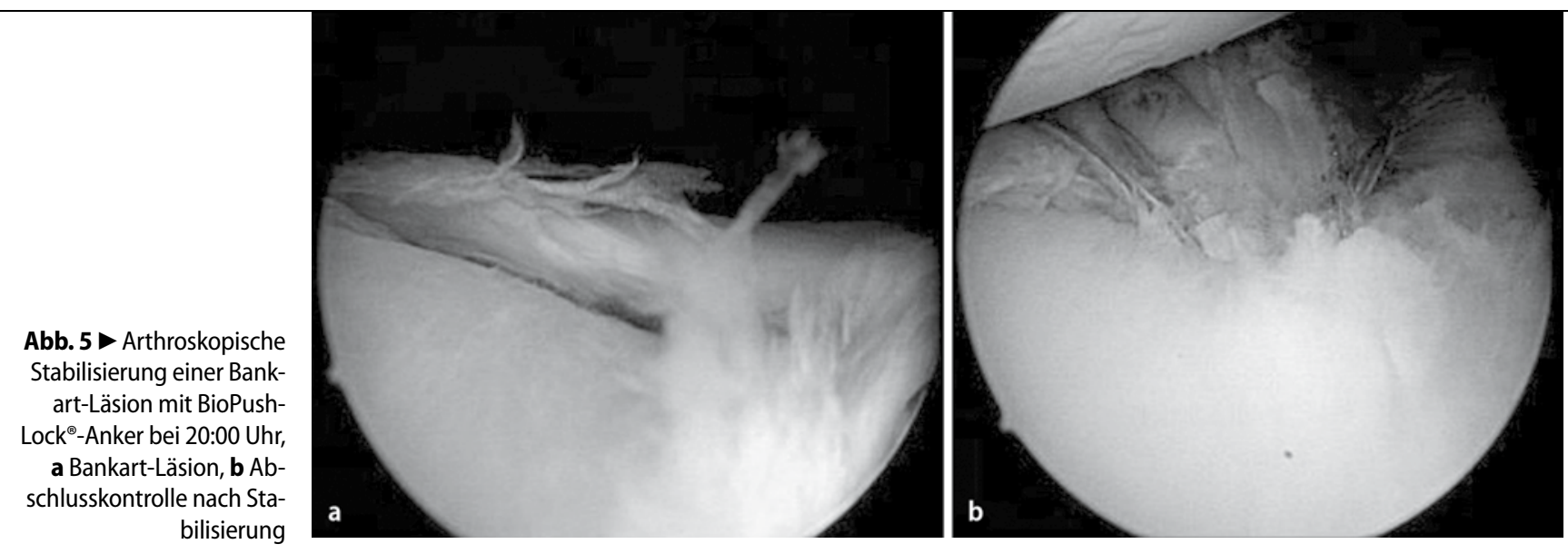

wurde diese Technik durch Verwendung von Nahtankern, die in den Pfannenrand eingebracht werden.

\section{Offene vs. arthroskopische Bankart-Reposition}

Nach wie vor steht die Art der BankartReposition zur Diskussion. In den letzten 3 Jahren wurden diesbezüglich 3 größere Metaanalysen [9, 21, 25] publiziert, die die Ergebnisse aus kontrollierten, mehrarmigen, in der Regel prospektiven Studien zur Therapie traumatischer Schulterinstabilitäten zusammentrugen. Alle Arbeiten verglichen eine arthroskopische mit einer offenen Bankart-Reposition. Hierbei zeigte sich, dass hinsichtlich der Rezidivrate ein offenes Vorgehen günstiger zu sein scheint. Die Wahl der verwendeten arthroskopischen Stabilisierungsmethode (Fadenanker, bioresorbierbare Tacks oder transglenoidale Nahttechniken) schien dabei keinen Einfluss zu haben. Des Weiteren konnte in diesen Arbeiten eine raschere Arbeits- und Sportfähigkeit nach offener Bankart-Reposition nachgewiesen werden. Umgekehrt wiesen die arthroskopischen Verfahren, insbesondere unter Verwendung von Fadenankern, eine im Vergleich signifikant bessere Schulterfunktion bezüglich des Rowe-Scores auf. Die Rate der Komplikationen war bei beiden Verfahren gleich und zeigte keine signifikanten Unterschiede.

Somit scheint aufgrund der bisherigen Datenlage ein offenes Vorgehen bezüglich der Reluxationsrate nach wie vor der Goldstandard zu sein. Dennoch wurden in der Vergangenheit mehrere prospektive Studien publiziert, die für die Arthroskopie den offenen Verfahren vergleichbar gute Ergebnisse aufzeigten und eine ähnliche Re- zidivrate von $5-10 \%$ nach primär traumatischer Schulterluxation beschrieben [4, $6,8,16,20,23,24,37]$. Vorteilhaft ist u. a. auch deren deutlich geringere Zugangsmorbidität. Insbesondere die (teilweise) Ablösung der Subskapularissehne, verbunden mit unterschiedlichen Techniken der Refixierung, die bei allen offenen Verfahren notwendig ist, scheint eine postoperative Einschränkung des Schulterbewegungsumfangs nach sich zu ziehen. Zum anderen sind nach offener Stabilisierung in bis zu $23 \%$ Subskapularisinsuffizienzen zu beobachten $[4,30]$. In der Hand des Geübten scheint die arthroskopische Stabilisierung in Verbindung mit einem individuell angepassten inferioren Kapselshift darüber hinaus auch als Revisionseingriff eine ernst zu nehmende Alternative darzustellen $([19,35], \boldsymbol{\square}$ Tab. 7$)$.

\section{Kapselshift}

Die Notwendigkeit und die Größe eines Kapselshifts richten sich nach der zugrunde liegenden Pathologie. Eine primär traumatische Schulterinstabilität mit nur kleiner inferiorer Kapseltasche benötigt in der Regel keinen oder nur einen kleinen inferioren Kapselshift. Zeigt sich jedoch intraoperativ eine große inferiore Kapseltasche, sind u. U. ein inferiorer Kapselshift und damit verbunden eine Reduktion des Kapselvolumens für den Therapieerfolg entscheidend.

Arthroskopisch konnte dieser Kapselshift sehr gut in der Operationstechnik nach Caspari realisiert werden. Hierbei werden die refixierenden Fäden transglenoidal durchgezogen und dorsal auf der Muskelfaszie verknotet. Wiederholt wurde dieser Technik jedoch vorgeworfen, dass sich keine optimalen und dauer- haften Anpressdrücke des Kapsel-BandApparats realisieren lassen, da die Verknotung auf einer dynamischen und nicht rigiden Auflage erfolgt. Auch sei diese Technik sehr anspruchsvoll und führe nur in der Hand von Erfahrenen zu konstant guten und reproduzierbaren Operationsergebnissen.

Mit anderen arthroskopischen Operationsverfahren ist es bisher nicht möglich, einen arthroskopischen Kapselshift zu bewerkstelligen. Eine inferiore Fixierung des Kapsel-Band-Apparats bedarf mit den bisher zur Verfügung stehenden Nahtankern eines tiefen anterior-inferioren Zugangs (etwa 5:00 Uhr) [7]. Dieser ist risikobehaftet (insbesondere beim Rezidiveingriff) bezüglich der Verletzung neurovaskulärer Strukturen, technisch anspruchsvoll und sollte mit der Technik vertrauten Operateuren vorbehalten bleiben [26]. Über ein typisches ventrales Standardportal ist aufgrund der Winkelverhältnisse eine korrekte Platzierung von Nahtankern am ventralen inferioren Glenoid nicht möglich.

Fadenanker, insbesondere knotenlose Nahtanker, bieten den Vorteil, einen wesentlich höheren Anpressdruck zur Refixierung des Kapsel-Labrum-Komplexes erzielen zu können ( $\boldsymbol{\square}$ Abb.5). Auch sind sie in der Regel einfach und schnell $\mathrm{zu}$ applizieren.

Mit der Entwicklung knotenloser Anker ergaben sich neue Perspektiven, die Vorteile der Operationstechnik nach Caspari mit den Vorteilen der Ankertechnik zu kombinieren. So führen wir einen wie von Caspari beschriebenen inferioren Kapselshift durch, bei dem der ventrale und inferiore Kapsel-Labrum-Komplex vom Glenoid abgelöst und die Kapsel bei 
etwa 19:0o Uhr ca. 1,5-2 cm nach kaudal eingeschnitten werden. Ähnlich wie bei Caspari wird dieser abgelöste Kapsel-Labrum-Komplex mit mehreren Fäden (z. B. FibreWire ${ }^{\circledR}$ angeschlungen und nach kranial gezogen (Shift). Hierbei kommt es je nach Ausmaß der inferioren Inzision zu einem unterschiedlich starken Shift. Die Fäden werden jedoch nun nicht wie bei Caspari transglenoidal nach dorsal ausgeleitet, sondern alle durch einen knotenlosen Nahtanker (z. B. BioPushLock ${ }^{\circledast}$ ) gefädelt und mit diesem sicher bei etwa 4:30 Uhr fixiert.

\section{Fazit für die Praxis}

Die traumatische Schulterluxation weist insbesondere bei den jüngeren, männlichen Patienten eine ausgesprochen hohe Rezidivwahrscheinlichkeit nach konservativer Therapie auf. Diese lässt sich innerhalb der ersten 2 Jahre auf etwa $87 \%$ beziffern. Zur konservativen Therapie scheint eine Immobilisation in AuBenrotationsstellung des Schultergelenks vorteilhaft. Zur operativen Therapie konkurrieren offene und arthroskopische Verfahren. Der Goldstandard zur Refixierung des abgerissenen ventralen Kapsel-Labrum-Komplexes scheint nach wie vor die offene Bankart-Operation darzustellen. In der Hand des Geübten können jedoch arthroskopische Stabilisierungen gleichwertige Ergebnisse hinsichtlich der Rezidivrate (5-10\%) liefern. Sie überzeugen zusätzlich durch eine geringere Zugangsmorbidität und postoperativ bessere funktionelle Ergebnisse.

\section{Korrespondenzadresse}

\section{Dr. M. Jaeger}

Department Orthopädie und Traumatologie, Universitätsklinikum Freiburg, Hugstetter Straße 55, 79106 Freiburg martin.jaeger@uniklinik-freiburg.de

Interessenkonflikt. Der korrespondierende Autor gibt an, dass kein Interessenkonflikt besteht.

\section{Literatur}

1. Allmann KH, Schäfer O, Hauer M et al. (1999) Indirect MR arthrography of the unexercised glenohumeral joint in patients with rotator cuff tears. Invest Radiol 34: 435-140

2. Bankart BA (1923) Recurrent or habitual dislocation of the shoulder-joint. BMJ 2: 1132-1133
3. Baykal B, Sener S, Turkan H (2005) Scapular manipulation technique for reduction of traumatic anterior shoulder dislocations: experiences of an academic emergency department. Emerg Med J 22: 336-338

4. Bottoni CR, Smith EL, Berkowitz MJ et al. (2006) Arthroscopic versus open shoulder stabilization for recurrent anterior instability: a prospective randomized clinical trial. Am J Sports Med 34: 17301737

5. Calandra JJ, Baker CL, Uribe J (1989) The incidence of Hill-Sachs lesions in initial anterior shoulder dislocations. Arthroscopy 5: 254-257

6. Carreira DS, Mazzocca AD, Oryhon J et al. (2006) A prospective outcome evaluation of arthroscopic Bankart repairs: minimum 2-year follow-up. Am J Sports Med 34: 771-777

7. Davidson PA, Tibone JE (1995) Anterior-inferior (5 o'clock) portal for shoulder arthroscopy. Arthroscopy 11: 519-525

8. Fabbriciani C, Milano G, Demontis A et al. (2004) Arthroscopic versus open treatment of Bankart lesion of the shoulder: a prospective randomized study. Arthroscopy 20: 456-462

9. Freedman KB, Smith AP, Romeo AA et al. (2004) Open Bankart repair versus arthroscopic repair with transglenoid sutures or bioabsorbable tacks for recurrent anterior instability of the shoulder: a meta-analysis. Am J Sports Med 32: 1520-1527

10. Gerber C, Nyffeler RW (2002) Classification of glenohumeral joint instability. Clin Orthop Relat Res 400: 65-76

11. Glombik TM (2004) Instabile Schulter. Trauma Berufskrankh 6: 126-133

12. Habermeyer $P$, Magosch P, Lichtenberg S (2004) Schulterinstabilitäten. Klassifikation und Behandlung. Orthopäde 33: 847-873

13. Harryman DT, Sidles JA, Clark JM et al. (1990) Translation of the humeral head on the glenoid with passive glenohumeral motion. J Bone Joint Surg Am 72: 1334-1343

14. Hovelius $L$ (1987) Anterior dislocation of the shoulder in teen-agers and young adults. Five-year prognosis. J Bone Joint Surg Am 69: 393-399

15. Hovelius L, Eriksson K, Fredin H et al. (1983) Recurrences after initial dislocation of the shoulder. Results of a prospective study of treatment. J Bone Joint Surg Am 65: 343-349

16. Ide J, Maeda S, Takagi K (2004) Arthroscopic Bankart repair using suture anchors in athletes: patient selection and postoperative sports activity. Am J Sports Med 32: 1899-1905

17. Itoi E, Hatakeyama Y, Urayama M et al. (1999) Position of immobilization after dislocation of the shoulder. A cadaveric study. J Bone Joint Surg Am 81: 385-390

18. Itoi E, Sashi R, Minagawa H et al. (2001) Position of immobilization after dislocation of the glenohumeral joint. A study with use of magnetic resonance imaging. J Bone Joint Surg Am 83-A: 661667

19. Kim S-H, Ha K-I, Kim S-H (2002) Arthroscopic revision Bankart repair. Arthroscopy18: 469-482

20. Kim S-H, Ha K-I, Kim S-H (2002) Bankart repair in traumatic anterior shoulder instability. Arthroscopy 18: 755-763

21. Lenters TR, Franta AK, Wolf FM et al. (2007) Arthroscopic compared with open repairs for recurrent anterior shoulder instability. A systematic review and meta-analysis of the literature. J Bone Joint Surg Am 89: 244-254

22. Loew M, Thomsen M, Rickert M et al. (2001) [Injury pattern in shoulder dislocation in the elderly patient]. Unfallchirurg 104: 115-118
23. Marquardt B, Witt KA, Liem D et al. (2006) Arthroscopic Bankart repair in traumatic anterior shoulder instability using a suture anchor technique. Arthroscopy 22: 931-936

24. Mazzocca AD, Brown FM, Carreira DS et al. (2005) Arthroscopic anterior shoulder stabilization of collision and contact athletes. Am J Sports Med 33: $52-60$

25. Mohtadi NG, Bitar IJ, Sasyniuk TM et al. (2005) Arthroscopic versus open repair for traumatic anterior shoulder instability: a meta-analysis. Arthroscopy 21:652-658

26. Pearsall AW, Holovacs TF, Speer KP (1999) The low anterior five-o'clock portal during arthroscopic shoulder surgery performed in the beach-chair position. Am J Sports Med 27: 571-574

27. Perthes G (1906) Über Operationen bei habitueller Schulterluxation. Dtsch Z Chir 85: 199

28. Robinson CM, Howes J, Murdoch H et al. (2006) Functional outcome and risk of recurrent instability after primary traumatic anterior shoulder dislocation in young patients. J Bone Joint Surg Am 88: 2326-2336

29. Rowe CR, Patel D, Southmayd WW (1978) The Bankart procedure: a long-term end-result study. J Bone Joint Surg Am 60: 1-16

30. Sachs RA, Williams B, Stone ML et al, (2005) Open Bankart repair: correlation of results with postoperative subscapularis function. Am J Sports Med 33: 1458-1462

31. Schmal H, Strohm PC, Rosahl SK et al. (2006) [Rupture of the arteria subscapularis following reduction of an anterior shoulder dislocation]. Unfallchirurg 109: 153-155

32. Seybold D, Gekle C, Fehmer T et al. (2006) [Immobilization in external rotation after primary shoulder dislocation]. Chirurg 77: 821-826

33. Stimson LA (1900) An easy method of reducing dislocations of the shoulder and hip. Med Record 57: 356-357

34. Südkamp NP (2000) Verdrehtrauma der Schulter. Wann welche Diagnostik? Trauma Berufskrankh [Suppl 3] 2: S364-S369

35. Südkamp NP, Stolle S, Bock A et al. (2000) Die Revision nach initial arthroskopischer oder offener Schulterstabilisierung. Arthroskopie 13: 229-231

36. Tingart $M$, Bäthis $H$, Bouillon B et al. (2001) [Therapy of traumatic anterior shoulder dislocation: current status of therapy in Germany. Are there scientifically verified therapy concepts?]. Chirurg 72: 677-683

37. Tjoumakaris FP, Abboud JA, Hasan SA et al. (2006) Arthroscopic and open Bankart repairs provide similar outcomes. Clin Orthop Relat Res 446: 227232 